\title{
Quality Control of Antenna Alignment and Receiver Calibration Using the Sun: Adaptation to Midrange Weather Radar Observations at Low Elevation Angles
}

\author{
PATRICIA Altube \\ Meteorological Service of Catalonia, and Department of Astronomy and Meteorology, \\ University of Barcelona, Barcelona, Spain \\ JOAN BECH \\ Department of Astronomy and Meteorology, University of Barcelona, Barcelona, Spain \\ Oriol ARgemí AND TOMEU Rigo \\ Meteorological Service of Catalonia, Barcelona, Spain
}

(Manuscript received 13 June 2014, in final form 12 January 2015)

\begin{abstract}
A quality control method for combined online monitoring of weather radar antenna pointing biases and receiver calibration using solar signals detected by an operational radar is adapted for application to midrange radar data $(80-150 \mathrm{~km})$. As the original method was developed using long-range data, additional criteria based on robust statistical estimators are imposed in the sun signature detection and selection process, allowing to discard observations biased by ground clutter or precipitation and to remove very influential outliers. The validity ranges of the physical model describing the solar interferences detected by the scanning radar antenna are explicitly defined and an equation for estimation of the effective scanning width in reception is provided in a thorough theoretical derivation. The method proposed reveals its sensitivity to changes in the antenna pointing accuracy and receiver calibration when applied to operational data obtained with three C-band radars during one year. A comparative study on the goodness of fit between a three- and a five-parameter model highlights the effect on the stability and accuracy of the antenna and receiver parameters retrieved for each radar system, considering the dissimilar information content of the observations collected by each radar. The performance of the proposed methodology under the effects of the presence of ground clutter and radio local area network interferences is discussed in the results presented.
\end{abstract}

\section{Introduction}

The growing number of quantitative applications of weather radar observations has increased the demand for quality control and monitoring procedures during recent years. Improvements and new developments in techniques using radar data, such as quantitative precipitation estimation, very short-range precipitation forecasting (i.e., nowcasting), hydrological modeling, or data assimilation in NWP models, rely largely on the quality of the input radar data as discussed in Collier (2009) and Rossa

Corresponding author address: Patricia Altube, Meteorological Service of Catalonia, Berlin 38-48, 08029 Barcelona, Spain. E-mail:paltube@meteo.cat et al. (2010). Examples of weather radar quality control systems have been described by Lakshmanan et al. (2007), Michelson et al. (2004), Saltikoff et al. (2010), and Ośródka et al. (2014), while Friedrich et al. (2006) and Gourley et al. (2006) provided specific procedures for polarimetric radar data. Similarly, other quality control methods have been developed for particular applications such as quantitative precipitation estimates (Harrison et al. 2000; Joe 2011; Szturc et al. 2011) and Doppler radar wind profiles (Holleman 2005; Holleman et al. 2008).

Within this framework, it is not surprising that techniques to assess weather radar system performance have been developed, reviewed, and improved substantially in the last decade, as is the case with antenna alignment and receiver calibration. Indeed, after the first proposal 
by Whiton et al. (1976), the use of the sun as a reference radio source for offline inspection of weather radar system gain and antenna pointing accuracy is currently of widespread employment and has been extensively discussed in the literature-see, for instance, Frush (1984), Pratt and Ferraro (1989), and Eastment et al. (2001). Tapping (2001) introduced the $10.7-\mathrm{cm}$-wavelength solar radio emission measurements at the Dominion Radio Astrophysical Observatory (DRAO, Pentincton, British Columbia, Canada) and described their adaptation to other frequencies for the gain estimation of small beamwidth antennas when statically pointing at the sun. Leskinen et al. (2002) manually fitted a model for the power detected by the radar in scanning through the center of the sun to observations collected in an offline passive scan to estimate the antenna pointing bias and beamwidth.

Darlington et al. (2003) established criteria for automated identification, at long ranges, of solar signatures detected in weather radar volumetric scans. They showed that, through polynomial fits of the individual sun interferences, statistical information about the antenna pointing bias in azimuth could be retrieved on a regular basis. Holleman and Beekhuis (2004) reinforced the criteria for identification of the sun interferences and presented a fully automatic procedure for online and simultaneous monitoring of weather radar antenna alignment and receiver chain calibration. The method consists of fitting, to daily detected solar signatures, a theoretical model for the power of the solar signal detected by an operational scanning radar. The paper was the precursor of a series of further works by Huuskonen and Holleman (2007) and Holleman et al. (2010) in which the method was consolidated by addressing the difficulties in solar positioning due to the effect of atmospheric refraction and by a more detailed insight into the theoretical model. Further developments and applications of the technique are tackled in Muth et al. (2012), Frech (2009, 2013), and Huuskonen et al. (2014).

The objectives of the present paper are threefold: First, to adapt the methodology in Holleman and Beekhuis (2004) to midrange radar data $(80-150 \mathrm{~km})$, attending to the characteristics of the target radar network of the Meteorological Service of Catalonia (SMC); second, to review the theoretical model for solar interference observations to provide an explicit formal framework; and third, to comparatively examine the stability and accuracy of a three-parameter model fit in comparison to a full fiveparameter retrieval when applied to real observational data of varying quality and information content.

\section{Overview of the method}

Characteristic signatures related to the radio emission by the sun detected daily in weather radar scans are recognizable in the plan position indicator (PPI) displays as signals spanning all ranges in the direction of the sun. Online application of the sun interference method requires automatic detection of these solar artifacts in polar reflectivity data. Constant power over range and temporal continuity characteristics of the sun signal, as well as the relative position of the interference with respect to the sun, are the base for the derivation of criteria that enable automatic detection while discriminating other signals of similar nature (Darlington et al. 2003; Holleman and Beekhuis 2004). At lowest antenna elevations, sun signals may be affected by additional phenomena (e.g., rain, ground clutter) or other interferences of similar signature but of nonsolar origin may be present [e.g., radio local area network (RLAN) signals], posing additional difficulties in the detection and characterization. Following Huuskonen and Holleman (2007), the sun interference observations resulting from the detection process are input for a theoretical model. The proposed model is a 2D Gaussian function for the detected power dependent of the relative displacement between the antenna axis and the sun disk center. Hence, adequate quantitative typification of the observations is fundamental: the power of the interference, its time, and location. Inversion of the model yields an estimation of the antenna pointing bias and of the solar power at the top of the atmosphere (TOA) as estimated from radar measurements. Comparison of this estimation with the reference data from a solar observatory allows the assessment of the receiver calibration status.

\section{Data}

\section{a. XRAD}

The sun monitoring method has been implemented for three C-band (5.3-cm wavelength), single-polarization operative Doppler weather radars of the SMC weather radar network (the XRAD): Creu del Vent (CDV; $41.6^{\circ} \mathrm{N}, 1.4^{\circ} \mathrm{E} ; 825 \mathrm{~m} \mathrm{MSL}$ ), La Miranda (LMI; $41.1^{\circ} \mathrm{N}$, $0.9^{\circ} \mathrm{E} ; 910 \mathrm{~m} \mathrm{MSL}$ ), and Puig d'Arques (PDA; $41.9^{\circ} \mathrm{N}$, $3.0^{\circ} \mathrm{E} ; 542 \mathrm{~m} \mathrm{MSL}$ ). The three radars display similar technical characteristics, with nominal antenna beamwidths of $\Delta_{B}^{\mathrm{az}} \approx 1.10^{\circ}$ and $\Delta_{B}^{\mathrm{el}} \approx 1.20^{\circ}$ in the horizontal and vertical, respectively, given up to a precision of $\pm 0.05^{\circ}$. These radar systems perform on a 6-min basis, a longrange, single-PPI scanning task for fast surveillance purposes and a short-range multiple-PPI volumetric scan. Particular parameters of the scanning strategy in each case are described in Table 1. For application of the method on a daily basis, observations collected both in long- and short-range scans are used.

The sampling settings of the XRAD radars result in an azimuthal resolution of about $1^{\circ}$. Seen from the earth, 
TABLE 1. Scanning parameters of the XRAD radar systems for each of the tasks performed: antenna scan speed $\left(\Omega_{\text {scan }}\right)$, nominal angular resolution of scanned radials $\left(\Delta_{R}\right)$, PRF, maximum range $\left(R_{\max }\right)$, and nominal elevations of the PPI sweeps within the task.

\begin{tabular}{ccccccc}
\hline \hline Task & Radar & $\Omega_{\text {scan }}\left({ }^{\circ} \mathrm{s}^{-1}\right)$ & $\Delta_{R}\left({ }^{\circ}\right)$ & PRF $(\mathrm{Hz})$ & $R_{\max }(\mathrm{km})$ & Elevation $\left(^{\circ}\right)$ \\
\hline Long range & $\mathrm{CDV}$ & & & & & \\
& LMI & 24 & 1 & 450 & 250 & 0.6 \\
Short range & PDA & & & & & \\
& CDV & & & $1000 / 750$ & 150 & $0.6,0.8,1.0,1.3,1.7,2.0$, \\
& LMI & 24 & & $1150 / 862$ & 130 & $3.0,4.0,5.0,6.0,8.0,10.0$, \\
& PDA & & & $1150 / 862$ & 130 & $13.0,16.0,21.0,27.0$ \\
\hline
\end{tabular}

the radiowave solar disk subtends an angle of $\approx 0.57^{\circ}$ in diameter. Hence, usually no more than one or two radial rays are affected by the solar signal in each PPI sweep. In general, the number and distribution of the sun interferences occurring per day depends on the scanning strategy in combination with the current solar trajectory and speed across the local sky, the sensitivity of the radar, and the orography, among others.

\section{b. Reference solar flux}

The solar flux data measured at the DRAO and distributed by the National Research Council of Canada (Solar Radio Monitoring Program database, since 2004, available online at http://www.spaceweather.gc.ca/solarflux/sx-eng. $\mathrm{php}$ ) is used as reference for assessment of the receiverchain calibration.

The DRAO database provides the disk-integrated solar flux density at the TOA, measured at $10.7-\mathrm{cm}$ wavelength. The conversion of $10.7-\mathrm{cm}$ solar flux measurements to other radio frequencies relies on separation of the integrated flux density into a sum of two distinct components (Tapping 2001): a quiet-sun background component at the desired wavelength and a slowly varying component, which is shape stable to changes in wavelength and whose absolute value is determined through an appropriate scaling factor. The integrated solar flux density at $5 \mathrm{~cm}$, $S_{0, \text { ref }}$ given in solar flux units (SFU), may be estimated with an accuracy of $\approx 1 \mathrm{~dB}$ from the DRAO reference flux $\left(S_{10.7}\right)$ using tabulated values of the scaling factor and quiet-sun components:

$$
S_{0, \text { ref }}=0.71\left(S_{10.7}-64\right)+126 .
$$

For a direct comparison of the estimated $P_{\mathrm{TOA}}$ with the reference power, $P_{\mathrm{TOA}, \text { ref }}(\mathrm{dBm})$, multiplied by the receiver bandwidth, $\Delta \omega(\mathrm{Hz})$, and antenna effective area, $A_{\text {eff }}\left(\mathrm{m}^{2}\right)$, together with a change of units has to be applied to the reference solar flux:

$$
P_{\text {TOA,ref }}=10 \log _{10}\left(\frac{1}{2} \Delta \omega A_{\mathrm{eff}} S_{0, \text { ref }} 10^{-22}\right) .
$$

The effective collection area of the antenna is defined through the along-axis antenna gain $g$ and the radar wavelength $\lambda: A_{\text {eff }}=g \lambda^{2} / 4 \pi$ (Doviak and Zrnić 2006). The $1 / 2$ factor is introduced to consider that the radar is sensitive to horizontally polarized radiation only, while the sun is an unpolarized source.

\section{Sun interference identification and characterization}

On a daily basis, the reflectivity data in output polar files is read and analyzed radial by radial in search of sun interferences. In the following, a detailed description of the procedure and criteria for automatic detection and characterization of sun interferences is given.

\section{a. Identification}

Originally, the sun interference detection algorithm was designed for application to radar scans reaching ranges beyond $200 \mathrm{~km}$. A radial ray with a reflectivity value in all or most of its bins at long ranges is a main signature for identification of a solar interference (Huuskonen and Holleman 2007) because at those ranges the detected signal is unlikely to come from precipitation or ground clutter echoes, due to the height the radar beam achieves. The choice of an appropriate minimum range threshold that ensures the absence of precipitation echoes depends on the elevation of the scan and is conditioned by the maximum range reached by the system. In typical midlatitude storms, echo heights can exceed $8 \mathrm{~km}$. For the XRAD radar located at lowest height above sea level (at $525 \mathrm{~m}$ ), the center of the beam exceeds, according to $k$-model beam propagation under standard conditions, an altitude of $8 \mathrm{~km}$ only for elevations of $3^{\circ}$ and above. In this situation, setting a minimum range-height criterion would imply to exclude scanned elevations up to $3^{\circ}$ from the analysis. However, solar observations collected at low elevations, which are more frequently sampled, may provide useful information for the model inversion.

In a first consideration, radial rays having a valid reflectivity value in $90 \%$ or more of the pixels located farther than $50 \mathrm{~km}$ away from the radar are selected, independently of their elevation. The $50-\mathrm{km}$ range 


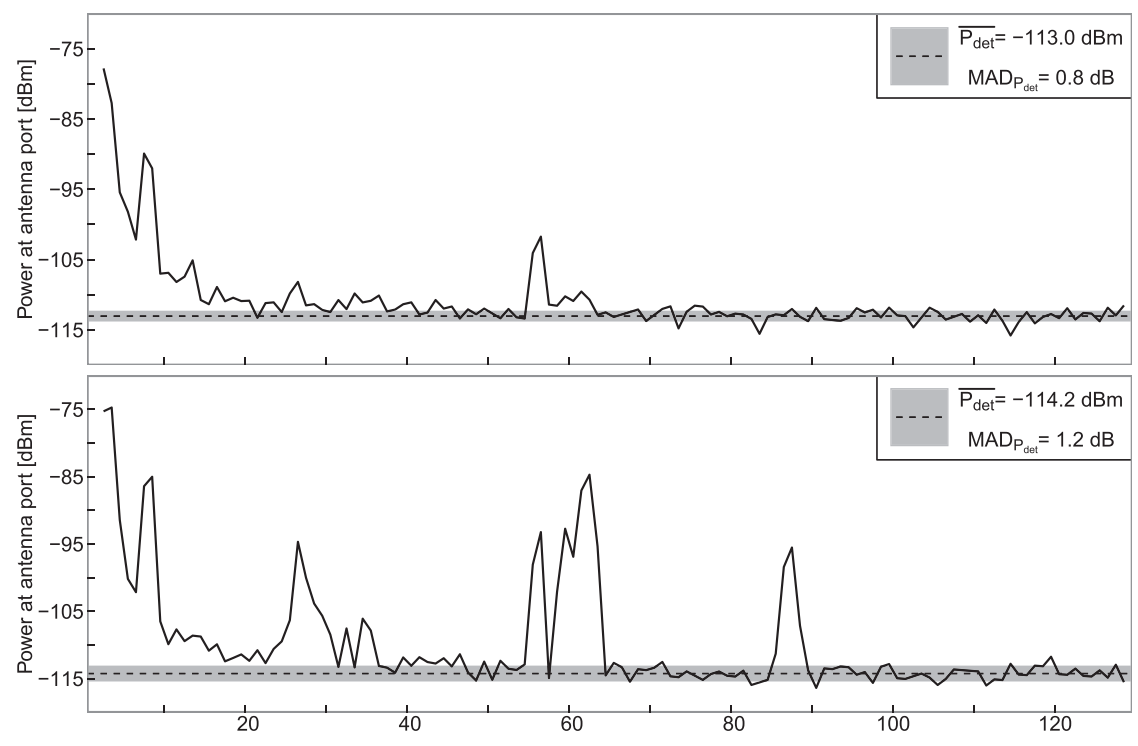

FIG. 1. An A-scope plot showing power at the antenna port after radar equation conversion $\left(P_{\text {det }}\right)$ for the PDA radar (16 Jun 2013). Two sun interference radials detected during sunset at different antenna positions are displayed: (top) 1902 UTC, $3^{\circ}$ elevation, and $299^{\circ}$ azimuth; and (bottom) $1907 \mathrm{UTC}, 2^{\circ}$ elevation, and $300^{\circ}$ azimuth. The dashed horizontal line indicates the median characteristic power of the interference $\left(\overline{P_{\mathrm{det}}}\right)$, and the shaded area encloses its $\sigma_{P \operatorname{det}}$ interval, both calculated only for bins at ranges beyond $80 \mathrm{~km}$.

threshold is chosen so that the areas close to the radar site most affected by ground clutter are skipped.

A definitive condition that discriminates sun interferences among the resulting set of selected radials is their relative position with respect to the sun; only interference radials located within $\pm 5^{\circ}$ from the actual solar position are considered. This criterion establishes the maximum antenna pointing offset detectable by the method. The relative position is calculated as the difference between the center of the interference radial and the center of the solar disk. Antenna readings at the start and end of the measurement are read from raw data files and transformed to radial center coordinates, $\left(\mathrm{az}_{R}, \mathrm{el}_{R}\right)$, and width, $\Delta_{R}$, for practicality. The solar position, $\left(\mathrm{az}_{S}, \mathrm{el}_{S}\right)$, relative to the radar site is calculated using astronomical equations (WMO 2008, chapter 7, 1.737-1.7-38) and local date, time, latitude, and longitude information. Given the maximum local speed of the sun across the sky, it has been estimated that the interference detection time needs to be accurate within $8-10$ s for an accuracy of $0.1^{\circ}$ in sun positioning.

Because of the effect of atmospheric refraction, the exoatmospheric or true sun elevation $\mathrm{el}_{S}$ may differ significantly from the solar elevation actually observed from the earth's surface, the apparent elevation $\mathrm{el}_{S}^{a}$ (Huuskonen and Holleman 2007). The refraction angle has been calculated through the theoretical formulas derived in Holleman and Huuskonen (2013), using the recommended value of $5 / 4$ for the $k$-model constant and a reference surface refractivity of 313 . Under these conditions and assuming an exponential humidity profile, a maximum accuracy error of $0.1^{\circ}$ in the refraction angle calculation is attributed (Holleman and Huuskonen 2013).

\section{b. Characterization of power}

Once a radial affected by a solar interference is identified, the reflectivity values $(\mathrm{dBZ})$ of each of its range pixels are individually back-converted to power $(\mathrm{dB} m)$ at the antenna entrance through application of the radar equation as implemented in the processor (Vaisala 2014). Considering all range bins farther away than $80 \mathrm{~km}$ from the radar site, the median value of their power $\overline{P_{\mathrm{det}}}$ is calculated and recorded as the characteristic power at the antenna port of the interference radial. The $80-\mathrm{km}$ range threshold is selected to minimize the possibility of obtaining a biased estimate of the detected power by the presence of ground clutter or precipitation. The choice of the median as estimator is also decisive in minimizing the effect of range bins with a power value deviating from the along-range constant value expected, as illustrated in the examples of Fig. 1.

At this point, a statistical deviation $\left(\sigma_{P \text { det }}\right)$ maximum threshold for the median power of the interference radial is established. Interference radials with an estimated $\sigma_{P \text { det }}$ of power below the threshold are kept for future processing steps. This is useful in bounding the error of the interference power and in discarding interferences strongly affected by ground clutter or 
precipitation echoes. As a robust estimator of $\sigma_{P \operatorname{det}}$, the median absolute deviation (MAD) of the bin ensemble is used, scaled so that it conforms to the underlying distribution (Reimann et al. 2008). If the bin-power deviations are derived only from random effects, then the $\sigma_{P \operatorname{det}}$ distribution is expected to be normal.

An analysis of the distribution of $\sigma_{P \operatorname{det}}$ values of sun interferences collected during 12 months leads to the choice of a static maximum threshold of $2 \mathrm{~dB}$. In the analysis, only continuous interference signatures located within $\pm 1^{\circ}$ from solar position have been considered. The bulk of the $\sigma_{P \text { det }}$ estimations lie, for all three radars, within an interval of $(1.0 \pm 0.5) \mathrm{dB}$ values. The density distributions appeared slightly skewed toward large $\sigma_{P \operatorname{det}}$ values and with several outlying observations. PDA radar revealed a larger number of outliers, probably related to the presence of ground echoes beyond $80 \mathrm{~km}$, as shown in Fig. 1, and to a greater influence of interfering RLAN signals.

\section{Sun interference model development}

In what follows, the power of the sun signal is modeled considering the radar antenna sensitivity pattern and the emission-reception scheme of the pulsed system while the antenna is in (azimuthal) motion.

For a single-reception sample, indexed by $j$, the fraction of solar power detected $p_{\mathrm{det}}^{j}$ is quantified by the convolution between the solar power pattern $S(\phi, \theta)$ and the antenna gain pattern $f\left(\phi-\phi_{j}^{0}, \theta-\theta^{0}\right)$ functions. Here, $(\phi, \theta)$ denote azimuthal and zenithal (spherical) coordinates and $\left(\phi_{j}^{0}, \theta^{0}\right)$ give the instantaneous position of the antenna radiation field center in a reference system with the origin in the radar and with its reference axis pointing at the sun disk center. For most cases, the solar power pattern function for radiowave emission may be modeled as a uniform disk of $\Delta_{S}$ diameter and $S_{0}$ integrated power flux density (Tapping 2001). In turn, the antenna sensitivity pattern function, assumed symmetric and normalized for unity axial gain, is represented as a 2D Gaussian, with $\Delta_{B}$ as the 3-dB beamwidth (Probert-Jones 1962).

Variation intervals of $(\phi, \theta)$ are small enough, with $\Delta_{S} \approx 0.57^{\circ}$, to assume the integration region in the convolution to be lying in a plane. Hence, plane trigonometry instead of a spherical one may be applied. The convolution is simplified considering sun and antenna pattern functions in a polar reference system (Holleman et al. 2010). A solution of the $2 \mathrm{D}$ convolution integral using elementary functions is not possible and a general solution is of the type

$$
p_{\mathrm{det}}^{j}=l_{\mathrm{gas}} p_{\mathrm{TOA}} f\left(\delta_{j} ; \Delta_{B}\right)
$$

where $\delta_{j}$ is the relative displacement between the sun disk center and the antenna axis; $p_{\mathrm{TOA}}$ is, in analogy to (2), the
TABLE 2. Antenna-sun convolution width $\Delta_{C}$ as a function of the antenna beamwidth $\Delta_{B}$. The $\Delta_{C}$ values are estimated by direct measurement of the half-peak width of the numerically computed convolution function. The computation considers a symmetric antenna pattern and a sun disk diameter of $0.57^{\circ}$.

\begin{tabular}{cc}
\hline \hline$\Delta_{B}\left(^{\circ}\right)$ & $\Delta_{C}\left(^{\circ}\right)$ \\
\hline 0.70 & 0.78 \\
0.75 & 0.83 \\
0.80 & 0.87 \\
0.85 & 0.92 \\
0.90 & 0.96 \\
0.95 & 1.01 \\
1.00 & 1.06 \\
1.10 & 1.15 \\
1.20 & 1.25 \\
1.30 & 1.34 \\
1.40 & 1.44 \\
1.50 & 1.54 \\
\hline
\end{tabular}

solar power at the TOA, and $l_{\text {gas }}$ is a factor accounting for the solar power path attenuation due to atmospheric gases. The latter may be estimated assuming a model atmosphere of constant density, so that the magnitude of the attenuation depends uniquely on the length of the solar energy path through the medium (Huuskonen and Holleman 2007).

Application of the adaptive Genz-Malik algorithm (Genz and Malik 1980) in the numerical integration of $f\left(\delta_{j} ; \Delta_{B}\right)$ for varying antenna beamwidths has shown that $p_{\text {det }}^{j}$ may be well approximated by a Gaussian function of $\Delta_{C}$ width for $\Delta_{B} \gtrsim 0.3^{\circ}$ :

$$
p_{\mathrm{det}}^{j}=l_{\mathrm{gas}} l_{0} p_{\mathrm{TOA}} \exp \left(-4 \ln 2 \frac{\delta_{j}^{2}}{\Delta_{C}^{2}}\right),
$$

with

$$
l_{0}=\frac{1}{\ln 2} \frac{\Delta_{B}^{2}}{\Delta_{S}^{2}}\left[1-\exp \left(-\ln 2 \frac{\Delta_{S}^{2}}{\Delta_{B}^{2}}\right)\right] .
$$

Estimates of the convolution width $\Delta_{C}$ have been obtained by direct measurement of the full width at half maximum (FWHM) of the numerically derived function. These estimates are given in Table 2 as a function of the antenna beamwidth. The accuracy of the estimates is within $\pm 0.005^{\circ}$ from the resolution of the numerical integration.

To account for the scanning motion of the antenna, the instantaneous azimuthal position of the antenna $\left(\phi_{j}^{0}\right)$ is rewritten as the sum of a fixed coordinate $\left(\phi^{0}\right.$; the central position of the axis within the whole scanned radial) and a moving coordinate $\left(\chi_{j}\right)$ :

$$
\delta_{j}^{2}=\left(\phi^{0}+\chi_{j}\right)^{2}+\theta^{02} .
$$


The power detected in the radial scan $p_{\text {det }}$ is calculated by the radar processor as the average of the power values $p_{\mathrm{det}}^{j}$ measured in the collection of consecutive samples performed while the antenna is in motion across a radial ray. When the azimuthal displacement of the antenna between samples is small compared to the total width of the radial $\left(\Delta_{R}\right)$, the variable $\chi_{j}$ in (6) may be assumed continuous and the pulse average approximated by an integral (Doviak and Zrnić 2006):

$p_{\mathrm{det}} \approx \frac{1}{\Delta_{R}} \int_{-\Delta_{R} / 2}^{\Delta_{R} / 2} p_{\mathrm{det}}^{j}(\chi) d \chi$, if $\Delta_{R} \gg \frac{\Omega_{\mathrm{scan}}}{\mathrm{PRF}}$

where $\Omega_{\text {scan }}$ is the antenna scan speed and PRF is the pulse repetition frequency.

The general solution of (7) with (4) and (6) is given in terms of the error function:

$p_{\text {det }}=l_{\text {gas }} l_{0} \frac{p_{\mathrm{TOA}}}{4} \frac{\Delta_{C}}{\Delta_{R}} \sqrt{\frac{\pi}{\ln 2}} \exp \left(-4 \ln 2 \frac{\theta^{02}}{\Delta_{C}^{2}}\right) F\left(\phi_{0} ; \Delta_{R}, \Delta_{C}\right)$,

with

$$
\begin{aligned}
F\left(\phi_{0} ; \Delta_{R}, \Delta_{C}\right) & =\left\{\operatorname{erf}\left[\frac{\sqrt{4 \ln 2}}{\Delta_{C}}\left(\phi^{0}+\frac{\Delta_{R}}{2}\right)\right]\right. \\
& \left.-\operatorname{erf}\left[\frac{\sqrt{4 \ln 2}}{\Delta_{C}}\left(\phi^{0}-\frac{\Delta_{R}}{2}\right)\right]\right\} .
\end{aligned}
$$

The overall shape of the nonelemental function $F\left(\phi_{0} ; \Delta_{R}, \Delta_{C}\right)$ is controlled by the $\Delta_{R} / \Delta_{C}$ ratio and closely resembles a Gaussian for values $\Delta_{R} / \Delta_{C} \lesssim 1.5$ (Blahak 2008). The model has a well-defined absolute maximum and based on the definition of the Gaussian FWHM, the effective scanning sun image width in reception $\left(\Delta_{C \text {,eff }}\right)$ may be estimated from the solution of the following transcendental equation, in analogy to section 7.8 in Doviak and Zrnić (2006):

$$
\left[F\left(\phi_{0} ; \Delta_{R}, \Delta_{C}\right)-\frac{2}{e} \operatorname{erf}\left(\sqrt{\ln 2} \frac{\Delta_{R}}{\Delta_{C}}\right)\right]_{\phi_{0}= \pm \phi_{0}^{\star}}=0,
$$

where $\phi_{0}^{\star}$ is the positive root of Eq. (10) and $\Delta_{C, \mathrm{eff}}=2 \sqrt{\ln 2} \phi_{0}^{\star}$.

Under these conditions, the solar power detected is given by

$$
p_{\text {det }}=l_{\text {gas }} l_{\text {scan }} p_{\mathrm{TOA}} \exp \left[-4 \ln 2\left(\frac{\theta_{0}^{2}}{\Delta_{C}^{2}}+\frac{\phi_{0}^{2}}{\Delta_{C, \text { eff }}^{2}}\right)\right],
$$

with

$$
l_{\text {scan }}=l_{0} \sqrt{\frac{\pi}{4 \ln 2}} \frac{\Delta_{C}}{\Delta_{R}} \operatorname{erf}\left(\sqrt{\ln 2} \frac{\Delta_{R}}{\Delta_{C}}\right) .
$$

The relative position coordinates available $(x, y)$ are expressed with respect to the radar's reference system:

$$
x=\mathrm{az}_{R}^{\mathrm{read}}-\mathrm{az}_{S} ; \quad y=\mathrm{el}_{R}^{\mathrm{read}}-\mathrm{el}_{S}^{a} .
$$

The transformation between $\left(\phi_{0}, \theta_{0}\right)$ and $(x, y)$ sets of coordinates would require spherical geometry calculations. In this regard, the formula $\phi_{0}=\cos \left(\mathrm{el}_{R}^{a}\right) x$ is accurate at least up to a $1 \%$, for elevations below $50^{\circ}$ and for $(\phi, \theta)$ varying within $\pm 5^{\circ}$, according to Blahak (2008). In addition, the effective width is assumed independent of elevation $\Delta_{C \text {,eff }} / \cos \left(\mathrm{el}_{R}^{a}\right) \approx \Delta_{C \text {,eff }}$, with an accuracy error below $1 \%$ for $\mathrm{el}_{R}^{a} \leq 8^{\circ}$. Accounting for the possibility of a systematic bias in antenna pointing $\left(x_{0}, y_{0}\right)$, all these considerations lead to the model equation solution proposed in Holleman et al. (2010):

$p_{\mathrm{det}}=l_{\mathrm{gas}} l_{\mathrm{scan}} p_{\mathrm{TOA}} \exp \left\{-4 \ln 2\left[\frac{\left(x-x_{0}\right)^{2}}{\Delta_{C, \mathrm{eff}}^{2}}+\frac{\left(y-y_{0}\right)^{2}}{\Delta_{C}^{2}}\right]\right\}$.

\section{Model inversion}

\section{a. Linearization}

The characteristic powers of the identified sun interference radials, each corrected for atmospheric attenuation, make up the input observations for fitting the model (14), constituting a (generally overdetermined) nonlinear inverse problem. In the case of a Gaussian function fit, a linear treatment and direct inversion by means of a linear least squares (LLS) procedure is possible (Caruana et al. 1986; Holleman and Beekhuis 2004), as long as the errors in the observations are multiplicative (Guo 2011). Logarithmic transformation of (14) and rearrangement of the terms yields a $2 \mathrm{D}$ parabolic model for the corrected power of the interference $(P, \mathrm{~dB} m)$ :

$P=P_{\mathrm{det}}-L_{\mathrm{gas}}=a_{x} x^{2}+a_{y} y^{2}+b_{x} x+b_{y} y+c$,

with

$$
\begin{gathered}
a_{x}=-\frac{B}{\Delta_{x}^{2}} ; \quad a_{y}=-\frac{B}{\Delta_{y}^{2}} ; \\
b_{x}=2 B \frac{x_{0}}{\Delta_{x}^{2}} ; \quad b_{y}=2 B \frac{y_{0}}{\Delta_{y}^{2}} ; \\
c=P_{0}-B\left(\frac{x_{0}^{2}}{\Delta_{x}^{2}}+\frac{y_{0}^{2}}{\Delta_{y}^{2}}\right),
\end{gathered}
$$


and

$$
B=40 \log _{10}(2) .
$$

The new model equation in (15) is linear in the parameters $a_{x}, a_{y}, b_{x}, b_{y}$ and $c$, which may be retrieved in an ordinary LLS fit. The original target parameters $\left(x_{0}, y_{0}, \Delta_{C}, \Delta_{C \text {,eff }}\right)$ are derived through reversion of (16)(18). The quadratic form in (15) represents a concave down parabola with its maximum located at the same position as the maximum of the original Gaussian function.

\section{b. Outlier removal}

Least squares estimates are highly sensitive to model outliers, that is, observations that do not appropriately follow the pattern of the theoretical model to be fitted. Such problematic observations may be present because of precipitation or ground echoes affecting the majority of the radial range bins considered or because of attenuation by precipitation, yielding an inaccurate estimation of the detected power. Also, continuous interferences of nonsolar origin, being close enough to the solar position, may go all through the identification process. In addition, an incorrect positioning of the sun relative to the interference may have a similar effect. In a less likely situation, model outliers may be present if the interference corresponds to a transient state of strong solar activity.

Here a simple, noniterative method for the removal of biased observations prior to inversion is proposed. The criterion is based on the assumption that, when the pointing bias is negligible, $\left(x_{0}, y_{0}\right) \lesssim 0.1^{\circ}$, the detected powers of the sun interference observations, corrected for the distance to the sun and for the atmospheric and scanning losses $P_{\text {corr }}$ should display a normal distribution with $\overline{P_{\mathrm{TOA}}}$ as its expected value. Following these assumptions, the $P_{\text {corr }}$ for each of the sun interferences is estimated from their detected power $P_{\text {det }}$ by reverse application of (14) in the form

$$
P_{\text {corr }}=P_{\text {det }}-L_{\text {gas }}-L_{\text {scan }}-\hat{L},
$$

where

$$
\hat{L}=-40 \log _{10}(2)\left(\frac{x^{2}}{\Delta_{C, \text { eff }}^{2}}+\frac{y^{2}}{\Delta_{C}^{2}}\right) .
$$

The median $\overline{P_{\text {corr }}}$ and its corresponding $\sigma_{P \text { corr }}$ estimator as derived from the MAD are computed for the target sun interference collection. Sun observations whose estimated $P_{\text {corr }}$ is not within the $2 \sigma_{P \text { corr }}$ interval around $\overline{P_{\text {corr }}}$ are considered outliers and rejected (Sprent and Smeeton 2007). The width of the limiting $\sigma$ interval might be adjusted to match the desired level of strength in the removal.
In case a significant antenna pointing bias is present, the basic assumption of a normal statistical distribution of the $P_{\text {corr }}$ is no longer reliable and its shape depends on the particular $(x, y)$ distribution of the dataset, as well as on the magnitude of the pointing bias. However, the use of robust estimators allows the application of the criterion even under such circumstances without loss of effectiveness in the removal of leverage outliers [i.e., those observations found at $(x, y)$ positions far from the mean $(\bar{x}, \bar{y})$ defined by the bulk of observations].

\section{c. Inversion approach}

The main target parameters to be retrieved in the inversion of the theoretical model in (14) are the solar power at the TOA $P_{\mathrm{TOA}}$; the systematic antenna pointing biases in azimuth and elevation $\left(x_{0}, y_{0}\right)$; and, optionally, the effective widths in azimuth $\Delta_{C \text {,eff }}$ and elevation $\Delta_{C}$, whose values can be estimated. Based on this parameter classification, two different approaches to the inversion are possible: a full-parameter quadratic function fit, considering all five parameters unknown as described before (5P model); and a three-parameter linear function fit with fixed $\Delta_{C}$ and $\Delta_{C, \text { eff }}$ (3P model), in which the quadratic terms in (15) are treated as constants for each of the observations.

Huuskonen and Holleman (2007) propose the latter approach as optimal for improvement of the stability of the fit. Since the quality of the observational data and its information content are variable (depending on the radar system, on environmental factors, and on the combination of the scanning strategy and solar motion), a lower number of model parameters are more likely to be independently determined by the collected data in the inversion. On the other hand, forcing the values of the widths may have an effect on the goodness of fit and hence in the accuracy of the parameter estimates. Also, Huuskonen et al. $(2010,2014)$ pointed out that the width estimates from the 5P model fit may give information on the radar system performance and on the pointing stability of the antenna.

\section{Results}

In the present section, selected results of the application of the method to a year (2013) of daily data are presented and briefly analyzed. In the particular case of the PDA radar, the application of the outlier removal procedure is assessed. The performance of the inversion for both the 5P and the 3P models is studied. The convolution widths corresponding to the nominal antenna beamwidths (section $3 \mathrm{a}$ ) are $\Delta_{C}^{\mathrm{az}} \approx 1.15^{\circ}$ and $\Delta_{C}^{\mathrm{el}} \approx 1.25^{\circ}$ from Table 2. Therefore, the effective widths set as fixed parameters in the 3P model fit result in $\Delta_{C \text {,eff }}=1.36^{\circ}$ and $\Delta_{C}=1.25^{\circ}$ after application of (10). 

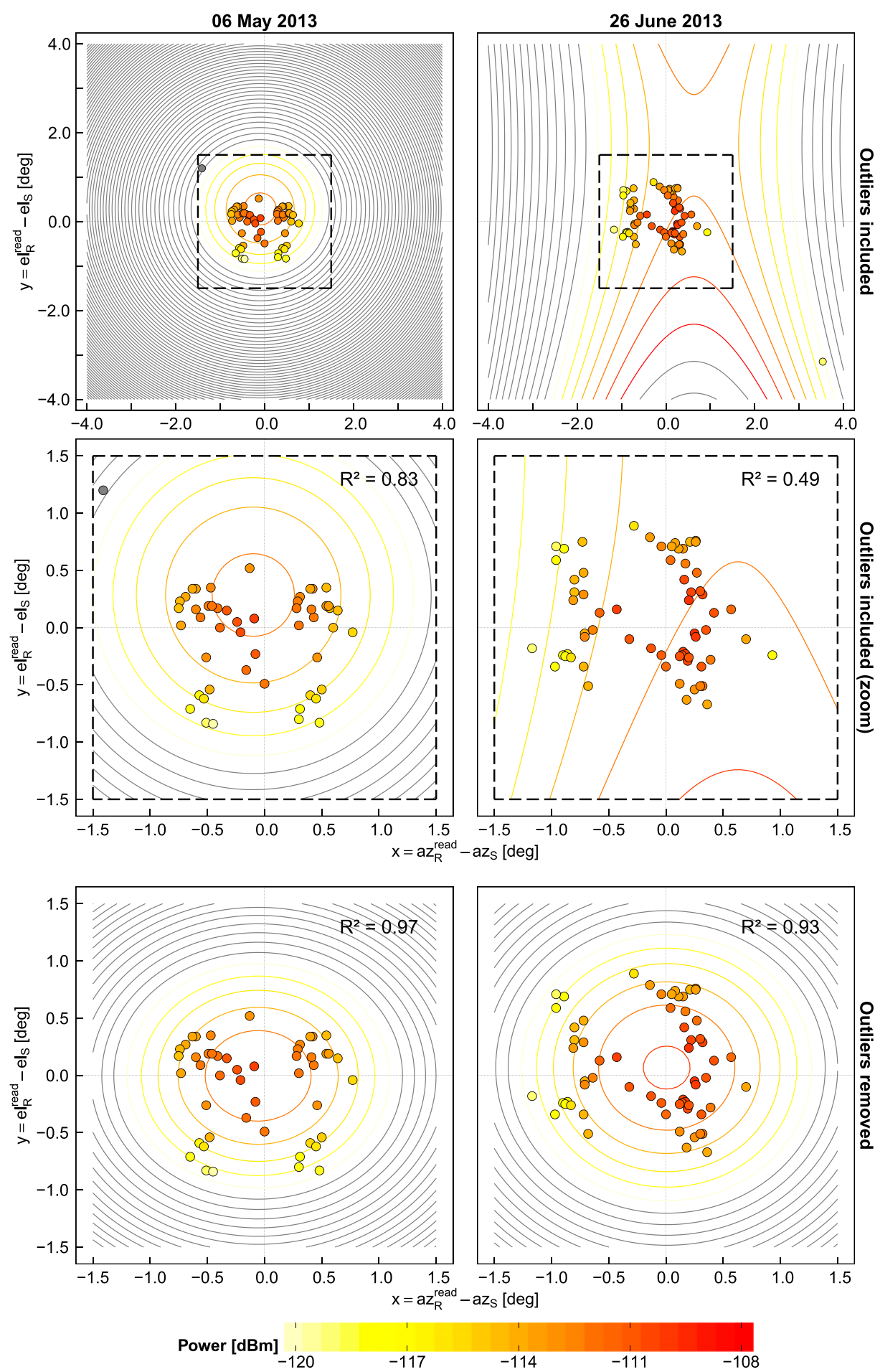

FIG. 2. Comparison of performance of the daily model fit to interference observations before and after outlier removal for the PDA radar (two cases: 6 May 2013 and 26 Jun 2013): (top) regular model fit using all identified interferences, (middle) regular model fit using all identified interferences-zoom into the framed region in the top panels, and (bottom) model fit after outlier removal criterion application. Observations are plotted by the relative position between the interference radial, as given by antenna readings, and the sun. Both the observations and the isolines of the resulting fitted model appear colorcoded as a function of the detected power, corrected for atmospheric attenuation. Adjusted $R$-squared values of the fit are given in each case. The resolution of the contour levels is $2 \mathrm{~dB}$. 

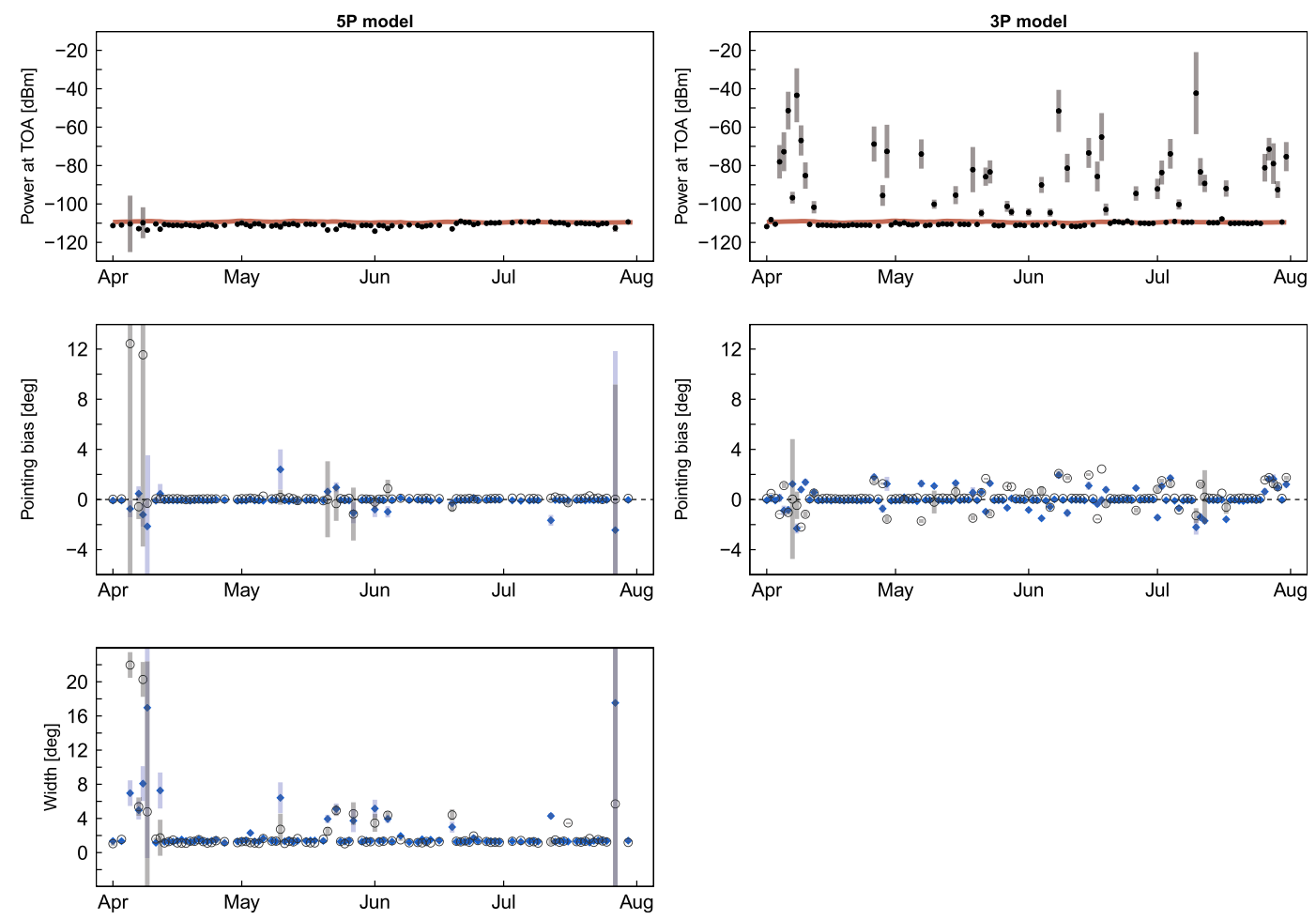

FIG. 3. Estimated values of the model parameters when the set of observations includes outliers, as obtained for the PDA radar from April to August 2013. Inversion results for the 5P and 3P models are compared for (top) the solar power at the TOA (black dots) in comparison with the reference from the DRAO (red solid line), (middle) the antenna pointing biases in azimuth (blue diamonds) and elevation (black circles), and (bottom) the sun image widths in azimuth (blue diamonds) and elevation (black circles). Bars indicate the precision errors arising uniquely from the inversion procedure.

Generally, no sun interferences are found for antenna elevations above $8^{\circ}$ and also the condition expressed in (7) is fulfilled for the XRAD radars, since the $1^{\circ}$ radial resolution is significantly higher than the maximum angular sampling interval of $0.032^{\circ}$ (from the corresponding $\Omega_{\text {scan }}$ and the minimum PRF in Table 1). Hence, the assumptions undertaken in the derivation of the theoretical model hold valid.

The goodness of fit is quantified through the rootmean-square deviation (RMSD) (i.e., the square root of the unbiased estimator for the variance of the fit residuals). The scale factor for the unbiased estimator is $\left(\mathrm{N}_{\text {intf }}-p-1\right)$, with $\mathrm{N}_{\text {intf }}$ as the number of observations and $p$ as the number of model parameters. In the optimal case, the RMSD should be of the order of the random error of the dataset. The lower limit for the RMSD is estimated in $\approx 0.3 \mathrm{~dB}$, as an approximation for the standard error of the median power $\overline{P_{\operatorname{det}}}$ in section $4 \mathrm{~b}$, calculated from the 2-dB statistical deviation threshold. As a means for assessing the comparison between inversion approaches, the adjusted $R$-squared value of the fit is used, since it quantifies the predictive power of the fitted model. The adjusted $R$-squared value is the relative decrease of the unbiased variance of the residuals (related to the RMSD as defined above) with respect to the unbiased total variance of the dataset.

\section{a. Puig d'Arques radar: Effect of leverage outliers}

The PDA radar has proven to be prone to presenting outlying observations, mainly due to contamination by emission from RLAN systems located close to the radar site. The interferences by these external electronic devices often show constancy and continuity characteristics similar to the solar ones. Daily, several constant and continuous interferences of presumed nonsolar nature are detected by PDA. These are easily recognizable because they generally occur at low elevation scans and because their positioning does not follow the solar trajectory. The sun proximity criterion is very effective in removing them but a few nonsolar interferences may still remain.

Observations detected far from the sun with a strong power signal have the potential to disturb the LLS fit the most and constitute leverage outliers. Figure 2 gives evidence of the effect of such type of observations on the result of the fit for two particular examples. When leverage outliers are present, the model fit does not 

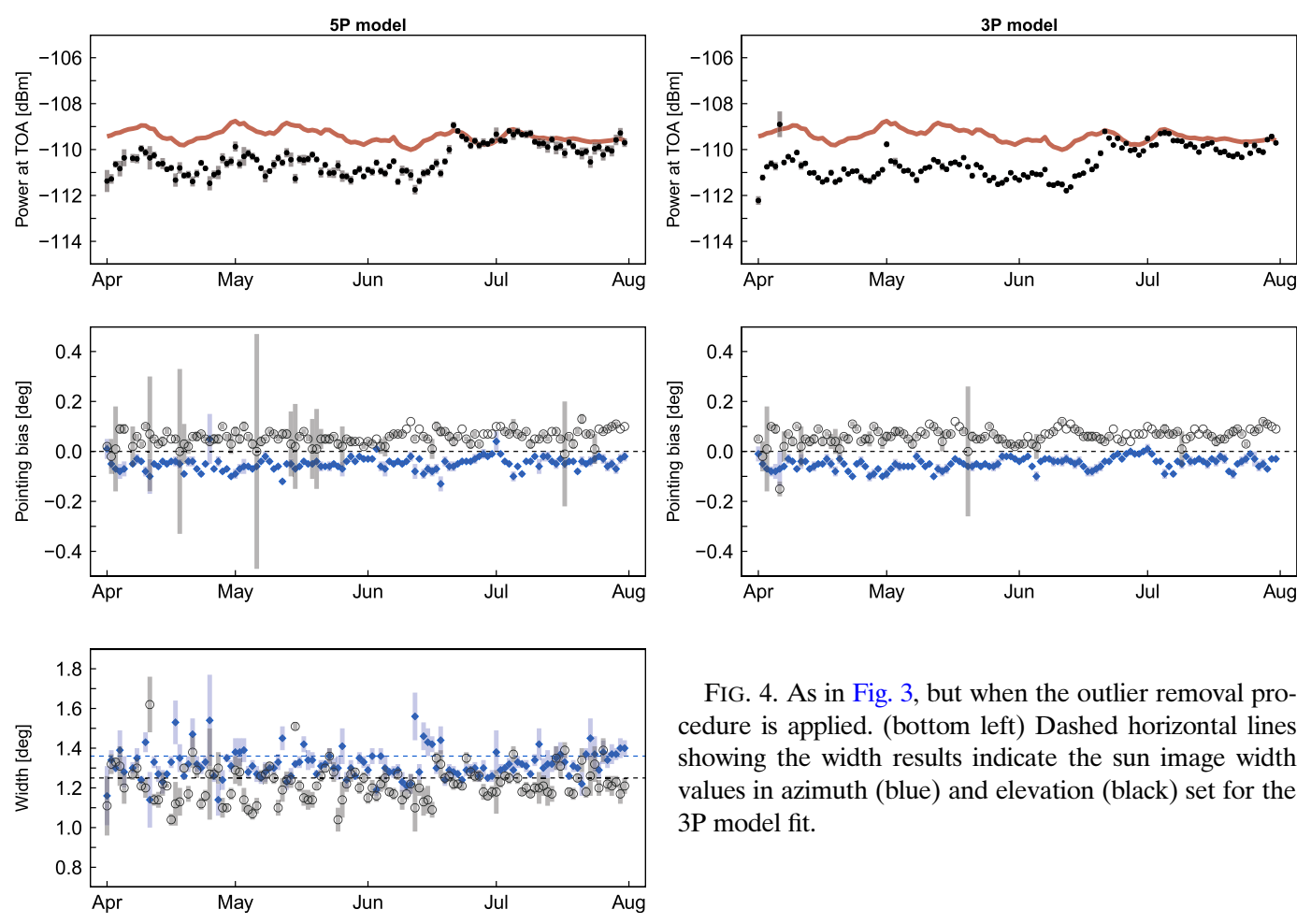

FIG. 4. As in Fig. 3, but when the outlier removal procedure is applied. (bottom left) Dashed horizontal lines showing the width results indicate the sun image width values in azimuth (blue) and elevation (black) set for the 3P model fit.

represent the bulk of the observations or even results in nonphysical solutions.

Figure 3 displays the day-to-day results of the sun interference model inversion for the data collected, before outlier removal was applied, during the period from April to July 2013. The inversion yielded nonphysical solutions for approximately $24.6 \%$ of the days considered. These failed fits appear as gaps in the $5 \mathrm{P}$ model time series plots. The impact of the outliers on the stability of the parameters (coherence and precision level) and on the convergence of the fit is remarkable. The presence of extreme outliers affects the estimates of all the model parameters considered. Power and pointing bias estimates in the $5 \mathrm{P}$ approach appear more

resilient to the effect outliers because the widths are additionally tuned for the model to fit the data and to reduce the cost function or RMSD. In these cases, the $3 \mathrm{P}$ model reacts with wild fluctuations both in power and in pointing. A part of these fluctuations is reflected in the width estimates of the 5P model. This is to be expected given the shape of the model function and the characteristics of the outliers-long distance and with high power values. Indeed, in extreme cases the 5P model fit leads to nonphysical solutions with negative width, as shown in Fig. 2 for 26 June 2013.

The outlier removal method allowed the retrieval of stable model estimates by both the 5P and 3P inversions, as shown in Fig. 4. Corresponding monthly statistics are

TABLE 3. Monthly statistics of PDA radar results from April to July 2013: maximum SNR estimated as the ratio between the maximum peak solar power (from the DRAO reference) and the minimum detectable power $P_{\mathrm{mds}}$, daily number of detected solar interference $\left(\mathrm{N}_{\text {intf }}\right)$, antenna pointing biases in azimuth $\left(x_{0}\right)$ and elevation $\left(y_{0}\right)$, peak solar power difference between the estimate and the reference from DRAO $(\Delta P)$, and sun image widths in azimuth $\left(\Delta_{C \text {,eff }}\right)$ and elevation $\left(\Delta_{C}\right)$. Given values correspond to the median and the median absolute deviation.

\begin{tabular}{lcccccccccc}
\hline \hline MNR & \multicolumn{1}{c}{ SNR } & & & & & & & & \\
Month & $(\mathrm{dB})$ & $N_{\text {intf }}$ & $x_{0}(3 \mathrm{P})\left(^{\circ}\right)$ & $x_{0}(5 \mathrm{P})\left(^{\circ}\right)$ & $y_{0}(3 \mathrm{P})\left(^{\circ}\right)$ & $y_{0}(5 \mathrm{P})\left({ }^{\circ}\right)$ & $\Delta P(3 \mathrm{P})(\mathrm{dB})$ & $\Delta P(5 \mathrm{P})(\mathrm{dB})$ & $\Delta_{C, \text { eff }}\left({ }^{\circ}\right)$ & $\Delta_{C}\left({ }^{\circ}\right)$ \\
\hline Apr & 9.4 & $44 \pm 8$ & $-0.06 \pm 0.03$ & $-0.05 \pm 0.03$ & $0.05 \pm 0.03$ & $0.05 \pm 0.03$ & $-1.57 \pm 0.24$ & $-1.33 \pm 0.30$ & $1.31 \pm 0.06$ & $1.21 \pm 0.12$ \\
May & 9.6 & $51 \pm 10$ & $-0.06 \pm 0.01$ & $-0.06 \pm 0.01$ & $0.06 \pm 0.03$ & $0.05 \pm 0.03$ & $-1.69 \pm 0.19$ & $-1.45 \pm 0.33$ & $1.31 \pm 0.04$ & $1.24 \pm 0.07$ \\
Jun & 9.2 & $57 \pm 9$ & $-0.04 \pm 0.02$ & $-0.04 \pm 0.03$ & $0.06 \pm 0.02$ & $0.06 \pm 0.02$ & $-1.44 \pm 0.37$ & $-1.08 \pm 0.54$ & $1.29 \pm 0.04$ & $1.21 \pm 0.07$ \\
Jul & 8.1 & $47 \pm 16$ & $-0.04 \pm 0.01$ & $-0.04 \pm 0.01$ & $0.07 \pm 0.03$ & $0.08 \pm 0.03$ & $-0.30 \pm 0.24$ & $-0.24 \pm 0.28$ & $1.33 \pm 0.06$ & $1.21 \pm 0.06$ \\
\hline
\end{tabular}



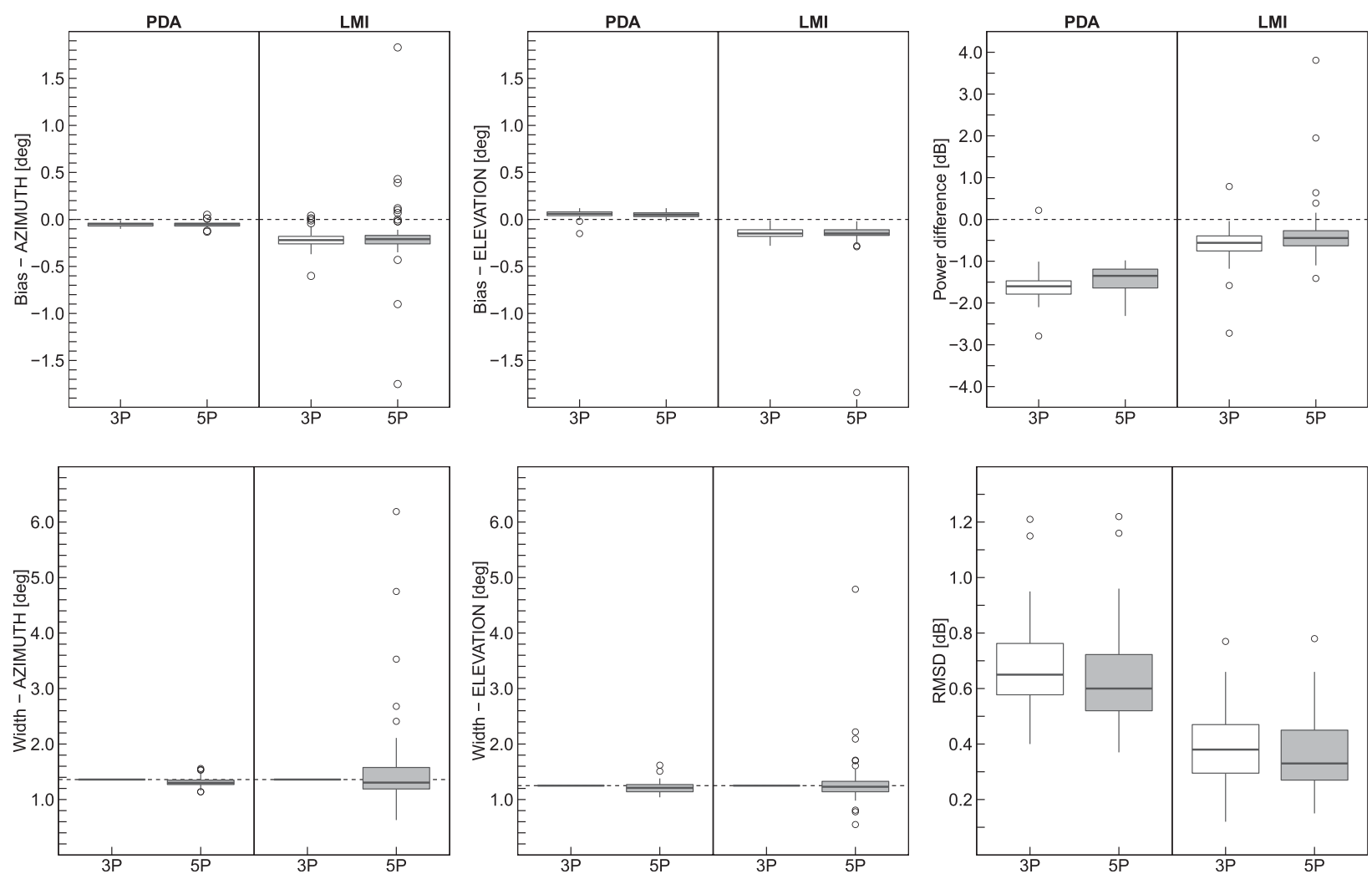

FIG. 5. Distributions of retrieved model parameters and RMSD comparing the 5P and 3P model fits for both PDA and LMI radars (from 1 Apr to 19 Jun 2013 and from 20 Sep to 9 Dec 2013, respectively). The boxes enclose the 1Q-3Q interquartile range, and the black solid line within the boxes highlights the median value. Outliers are displayed as circles outside the 1.5 times the interquartile range indicated by the whiskers. The sun image width values set for the 3P model fit are indicated by horizontal dashed lines.

given in Table 3. Estimates of $P_{\mathrm{TOA}}$ and the DRAO reference data displayed a good match between their respective trends. The difference of about $-1.5 \mathrm{~dB}$ between their values was corrected in a technical in situ recalibration of the radar receiver on 20 June. The intervention is reflected in the abrupt change of the estimated $P_{\mathrm{TOA}}$, indicating great sensitivity in the monitoring method.

\section{b. La Miranda and Puig d'Arques radars: Effect of the information content}

Figure 5 summarizes the statistics of LMI and PDA monitoring results for selected periods of 2013 for which the calibration status of both radars may be assumed invariant. These statistics serve to compare the performance of the 5P and 3P model fits under two situations for which the information contained in the set of daily observations is different.

On a daily basis, PDA detected 40-70 solar interferences (see Table 3), homogeneously distributed within distances up to $\pm 1^{\circ}$ and $\pm 0.8^{\circ}$ from the solar disk center in azimuth and elevation, respectively. These datasets are appropriate for the retrieval of the sun image widths. The widths estimated by the $5 \mathrm{P}$ model fit displayed a variability confined within $\pm 0.1^{\circ}$, indicative of a good and stable quality in the data collection by the radar. The comparison of the adjusted $R$-squared values for the two fitting strategies shown in Fig. 6 shows that 5P model estimates performed better in explaining the datasets, yielding stable and close to $1 R$-squared values. The 3P model inversion leads to very unstable and considerably lower $R$-squared values that point to some difficulty in the model for reproducing the data variability. In effect, the $P_{\mathrm{TOA}}$ estimates by the $3 \mathrm{P}$ model showed a systematic difference of about $-0.3 \mathrm{~dB}$ with respect to the $5 \mathrm{P}$ model, attributed to an overestimation of the fixed widths in azimuth and elevation as inferred from the 5P width results in Fig. 5. The inaccuracy of the fixed widths is about $-0.05^{\circ}$ and is attributed to the precision of the nominal antenna beamwidth values available.

Because of a lower sensitivity of the receiving system, often not enough sun daily interferences for the model inversion were detected in the case of LMI radar. The minimum detectable power $\left(P_{\mathrm{mds}}\right)$ is determined by the response curve of the radar receiver and the magnitude 


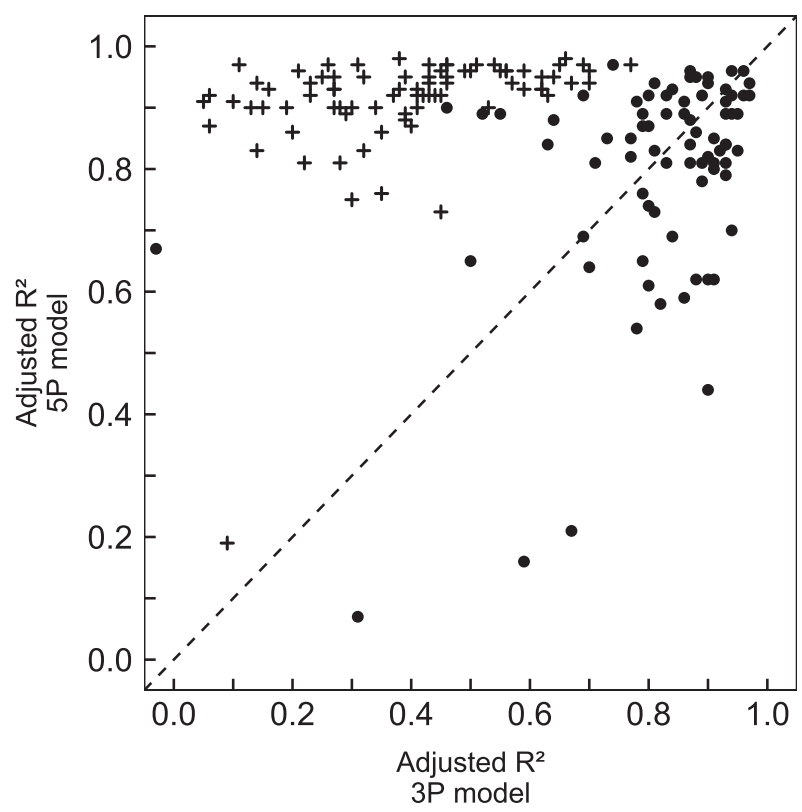

FIG. 6. Scatterplot of the adjusted $R$-squared value of fit comparing the $5 \mathrm{P}$ and the $3 \mathrm{P}$ models for PDA (crosses) and LMI (dots) radars (from 1 Apr to 19 Jun 2013 and from 20 Sep to 9 Dec 2013, respectively).

of the (signal + noise)-to-noise ratio threshold filter (LOG filter) (Vaisala 2014), which is set at $+4.5 \mathrm{~dB}$ with respect to the average noise power. The combination of these factors resulted in values of $P_{\mathrm{mds}}$ for LMI that were only slightly lower than the registered solar powers at the TOA, as expressed by low peak power signal-to-noise ratio (SNR) values in Table 4.

During the second half of 2013, LMI collected between 10 and 20 interferences per day, reaching 20-30 interferences per day from October on, when the solar activity showed a continuous increase. Even under these circumstances, the receiving system detected only solar interferences with a high power for which the antenna was closely pointing to the center of the sun disk (not farther than $\pm 0.5^{\circ}$ in azimuth and elevation). The information content in such a distribution of the observations is not appropriate for precise retrievals of the image width by the 5P model, especially in the azimuthal direction. The inverse problem is ill posed for the estimation of the widths, and their estimates have a strong dependence on the daily variable setting and precision of the observations available for the fit. Retrieved azimuthal widths were unstable with a day-to-day variability of $\pm 0.3^{\circ}$, as confirmed by the deviations shown in Table 4 . To a lesser degree, the lack of information also affected the variability of pointing bias estimates in the azimuthal direction both for the 5P and $3 \mathrm{P}$ inversions. As a result of the lower number of observations and their distribution, the RMSD values for LMI were significantly lower than for PDA and close to the lower limit of $0.3 \mathrm{~dB}$. The lack of information for the $5 \mathrm{P}$ model retrieval is confirmed in the analysis of the adjusted $R$-squared value of the fit for LMI (Fig. 6); despite the generally lower RMSD values shown in Fig. 5, the predictive power of the 5P model was not improved with respect to the $3 \mathrm{P}$ model fit, as indicated by the similar $R$-squared values.

The antenna pointing bias estimates for LMI indicated median errors around $-0.22^{\circ}$ in azimuth and around $-0.15^{\circ}$ in elevation. These exact pointing biases were verified in a later in situ laser tracker antenna alignment test and attributed to a malfunction of the azimuth encoder.

\section{c. Creu del Vent radar: Effect of radar system performance}

Figure 7 shows the elevation antenna pointing bias and width results for CDV from May to October 2013. Corresponding monthly statistics are given in Table 5. During the studied period, the radar system went through three different performance stages that could be identified in the results of application of the monitoring method. Relevant dates delimiting the stages are indicated by dashed vertical lines.

Prior to a software upgrade on 13 June, CDV detected few interferences, generally less than 20 per day, often falling below 10, and with great variability from day to day (see Table 5). Moreover, the distribution of relative positions of the interference was not uniform as expected. In particular, no interferences were detected for a stripe spanning $0.2^{\circ}$ in azimuth, causing a remarkable data void region. This fact is well illustrated in Fig. 8, showing the data distribution before and after the software upgrade. The severe lack of information for the retrieval leads to very variable elevation bias and width

TABLE 4. As in Table 3, but for the LMI radar from September to December 2013.

\begin{tabular}{ccccccccccc}
\hline \hline Month & $\begin{array}{l}\text { SNR } \\
(\mathrm{dB})\end{array}$ & $N_{\text {intf }}$ & $x_{0}(3 \mathrm{P})\left({ }^{\circ}\right)$ & $x_{0}(5 \mathrm{P})\left({ }^{\circ}\right)$ & $y_{0}(3 \mathrm{P})\left(^{\circ}\right)$ & $y_{0}(5 \mathrm{P})\left({ }^{\circ}\right)$ & $\Delta P(3 \mathrm{P})(\mathrm{dB})$ & $\Delta P(5 \mathrm{P})(\mathrm{dB})$ & $\Delta_{C, \mathrm{eff}}\left({ }^{\circ}\right)$ & $\Delta_{C}\left({ }^{\circ}\right)$ \\
\hline Sep & 3.8 & $10 \pm 2$ & $-0.22 \pm 0.10$ & $-0.24 \pm 0.09$ & $-0.13 \pm 0.03$ & $-0.12 \pm 0.06$ & $-0.08 \pm 0.61$ & $-0.01 \pm 0.45$ & $1.15 \pm 0.16$ & $1.17 \pm 0.20$ \\
Oct & 5.5 & $16 \pm 6$ & $-0.20 \pm 0.08$ & $-0.19 \pm 0.09$ & $-0.16 \pm 0.05$ & $-0.16 \pm 0.04$ & $-0.52 \pm 0.29$ & $-0.34 \pm 0.30$ & $1.36 \pm 0.36$ & $1.19 \pm 0.13$ \\
Nov & 5.6 & $19 \pm 4$ & $-0.22 \pm 0.04$ & $-0.23 \pm 0.04$ & $-0.14 \pm 0.05$ & $-0.13 \pm 0.05$ & $-0.55 \pm 0.25$ & $-0.44 \pm 0.22$ & $1.29 \pm 0.18$ & $1.29 \pm 0.13$ \\
Dec & 5.7 & $25 \pm 6$ & $-0.24 \pm 0.04$ & $-0.23 \pm 0.04$ & $-0.14 \pm 0.03$ & $-0.14 \pm 0.03$ & $-0.56 \pm 0.15$ & $-0.46 \pm 0.25$ & $1.24 \pm 0.15$ & $1.23 \pm 0.13$ \\
\hline
\end{tabular}



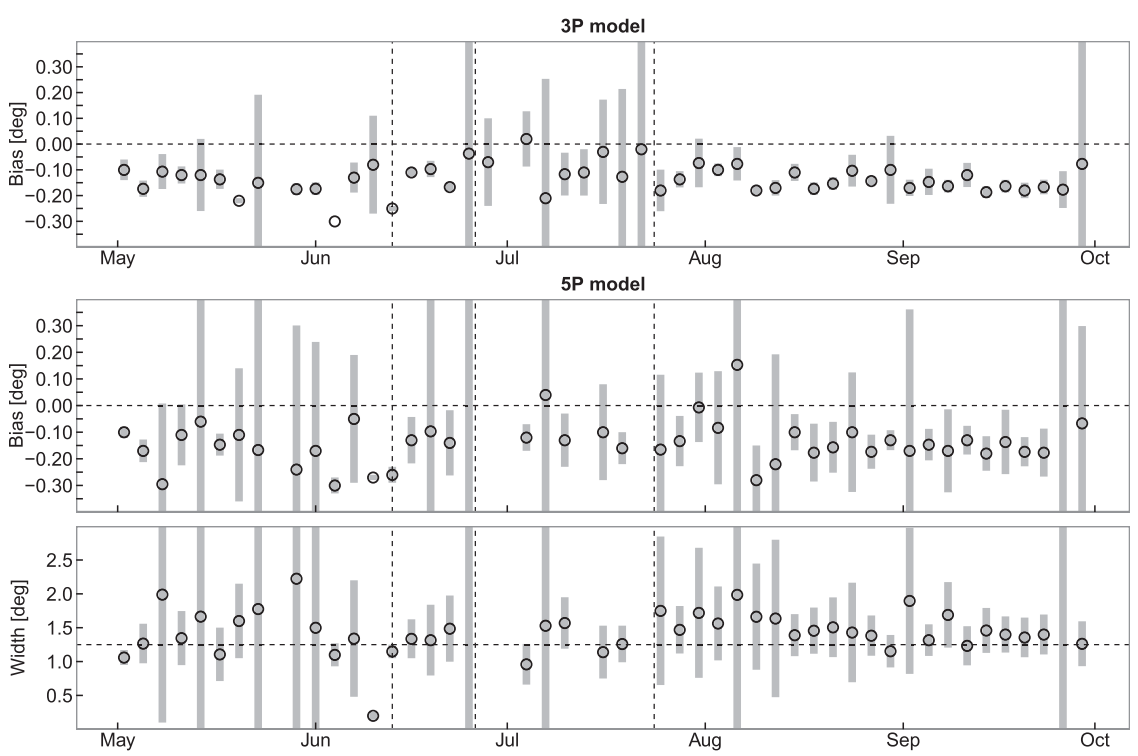

FIG. 7. Pointing bias and width estimates in elevation for the CDV radar from May to September 2013. Shown are 3-day mean values. Bars indicate the error of the mean as derived from the uncertainties of the estimates within the 3-day period.

estimates with large uncertainties. The anomaly in the data distribution was solved in the software upgrade, suggesting a problem in the processing module.

On 26 June, oscillations in the transmitter amplification operation were detected and this situation persisted until 24 July, when the traveling wave tube (TWT) was replaced. Throughout this period, the unstable operation of the TWT may have affected the noise figure and hence the sensitivity of the system. The daily number of detected interferences was continuously below 10 and the quality of the solar power observations, those being close to the noise level, deteriorated. When the number of observations allowed the sun interference model inversion, all parameter estimates were variable with large uncertainties and the 5P fit often returned nonphysical solutions. After the TWT change, the number of solar interferences increased above 20 and the improvement in the day-to-day stability of the model parameter results was significant. The variability of the estimates evidenced after 24 July has been attributed to system sensitivity, since no interferences were detected at relative distances beyond about $\pm 0.7^{\circ}$ in azimuth and about $\pm 0.5^{\circ}$ in elevation.

\section{Conclusions}

The online sun detection method for combined monitoring of weather radar antenna pointing biases and receiver calibration is adapted to midranges and applied to the weather radar network of the SMC. Originally, the method was designed for operation with long-range radar scans and the use of shorter range scans poses additional difficulties with the detection and characterization of sun interferences. Hence, given the maximum ranges reached by the target radar systems and to avoid sun observations biased by ground clutter or precipitation, an additional maximum along-range statistical deviation threshold for the power of the interference is imposed in the detection process. The threshold is set to $2 \mathrm{~dB}$, attending to the typical deviations presented by sun interference observations as derived from an ad hoc analysis.

TABLE 5. As in Table 3, but for the CDV radar from May to September 2013.

\begin{tabular}{|c|c|c|c|c|c|c|c|c|c|c|}
\hline Month & $\begin{array}{l}\text { SNR } \\
(\mathrm{dB})\end{array}$ & $N_{\text {intf }}$ & $x_{0}(3 \mathrm{P})\left(^{\circ}\right)$ & $x_{0}(5 \mathrm{P})\left({ }^{\circ}\right)$ & $y_{0}(3 \mathrm{P})\left({ }^{\circ}\right)$ & $y_{0}(5 \mathrm{P})\left({ }^{\circ}\right)$ & $\Delta P(3 \mathrm{P})(\mathrm{dB})$ & $\Delta P(5 \mathrm{P})(\mathrm{dB})$ & $\Delta_{C, \text { eff }}\left({ }^{\circ}\right)$ & $\Delta_{C}\left({ }^{\circ}\right)$ \\
\hline May & 8.4 & $16 \pm 9$ & $-0.08 \pm 0.03$ & $-0.07 \pm 0.03$ & $-0.15 \pm 0.07$ & $-0.16 \pm 0.09$ & $-0.53 \pm 0.39$ & $-0.54 \pm 0.73$ & $1.39 \pm 0.19$ & $1.33 \pm 0.40$ \\
\hline Jun & 8.1 & $14 \pm 13$ & $-0.04 \pm 0.04$ & $0.06 \pm 0.06$ & $-0.11 \pm 0.08$ & $-0.15 \pm 0.10$ & $-1.75 \pm 0.24$ & $-1.83 \pm 0.22$ & $1.41 \pm 0.21$ & $1.34 \pm 0.28$ \\
\hline Jul & 8.2 & $10 \pm 6$ & $-0.03 \pm 0.10$ & $-0.05 \pm 0.07$ & $-0.10 \pm 0.06$ & $-0.12 \pm 0.06$ & $-2.33 \pm 1.33$ & $-1.31 \pm 1.90$ & $1.56 \pm 0.42$ & $1.56 \pm 0.55$ \\
\hline Aug & 7.5 & $26 \pm 3$ & $-0.08 \pm 0.04$ & $-0.07 \pm 0.03$ & $-0.13 \pm 0.06$ & $-0.14 \pm 0.09$ & $0.88 \pm 0.56$ & $0.53 \pm 0.74$ & $1.43 \pm 0.16$ & $1.46 \pm 0.31$ \\
\hline Sep & 7.1 & $23 \pm 4$ & $-0.10 \pm 0.03$ & $-0.10 \pm 0.06$ & $-0.16 \pm 0.07$ & $-0.15 \pm 0.07$ & $-0.73 \pm 0.33$ & $-0.85 \pm 0.37$ & $1.46 \pm 0.29$ & $1.44 \pm 0.19$ \\
\hline
\end{tabular}




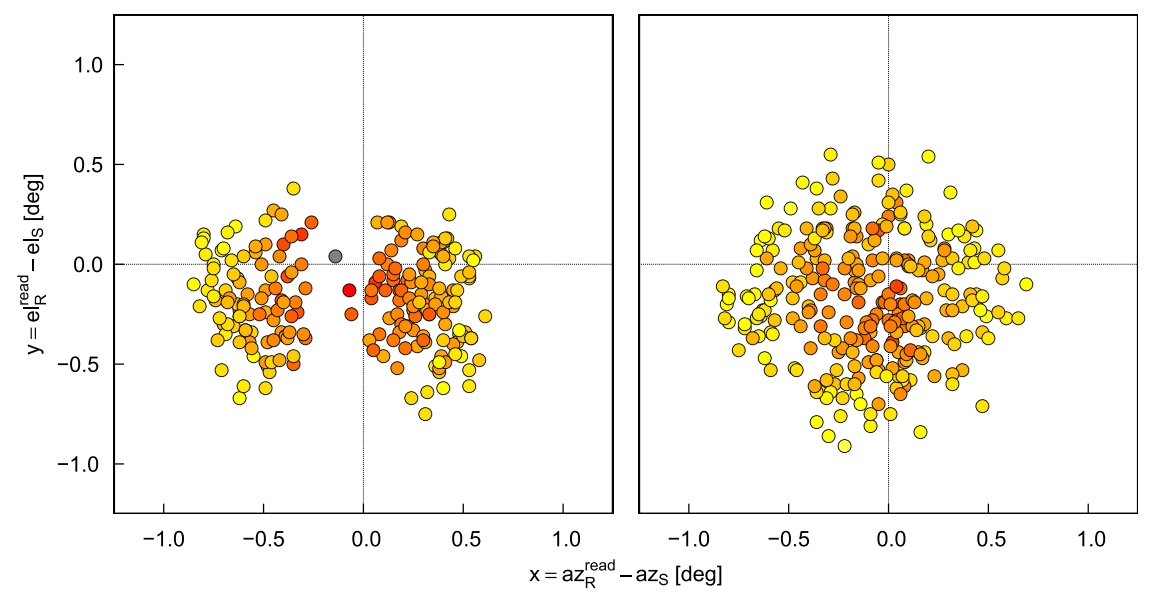

FIG. 8. Relative positions of sun interferences collected by the CDV radar in (left) May 2014, before a software upgrade; and (right) August 2013, after completing the upgrade. Interferences are color-coded as in Fig. 2.

A theoretical derivation of the physical model for weather radar sun observations defines the model validity ranges based on system settings and provides a means for estimating the effective width in reception of a scanning antenna. In particular, the proposed Gaussian model adequately describes solar interferences detected by the scanning radar antenna, at elevations preferably below $10^{\circ}$, when the ratio between the radial resolution and the convolution width is below 1.5.

Prior to model inversion, application of a noniterative method based on robust statistical estimators proves very efficient for the removal of leverage model outliers in this case attributed to nonsolar, presumably RLAN, interferences. This, together with the aforementioned identification threshold, allows the application of the monitoring method to sun interferences found at low antenna elevations even when only data at relatively short ranges are available. When the antenna pointing errors are not significant, the criterion might be adjusted for rejection of subtle outliers, such as sun signals attenuated by rain or biased by ground or precipitation echoes, aiming for an improved accuracy in the model parameter retrieval.

The monitoring method is applied to a year of daily sun observations for three different weather radars. The dissimilar information content carried in the collection of daily sun interferences detected by the radars serves to evaluate the performance of a full fiveparameter retrieval in comparison to a fixed threeparameter model fit. When the information content of the dataset used for the inversion is appropriate, the $5 \mathrm{P}$ approach comes out as the best for explaining the observations, with an optimal goodness of fit and yielding stable and precise parameter estimates. In this situation, the statistics of the retrieved widths effectively gives information about the quality in the data collection by the radar. Fixing the azimuth and elevation widths in the model fit smoothes the day-to-day fluctuations of the retrieved parameter values but may also introduce a systematic error, as a consequence of inaccuracies in the preliminary setting of the widths. However, when the dataset systematically lacks information for the retrieval of the widths-as seen in the cases of a limited sensitivity and of an anomalous data distribution-the day-to-day variability of the $5 \mathrm{P}$ model width estimates is dominated by the ill conditioning of the inverse problem. From the point of view of antenna alignment and receiver calibration status assessment, in these cases, application of the 3P model reduces the uncertainty and fluctuations of the estimates, without a significant loss of fit quality with respect to the $5 \mathrm{P}$ approach. For episodic observational data information and quality shortfalls (e.g., due to a particular combination of the scanning strategy and the solar motion or due to transient meteorological and atmospheric conditions), other strategies such as a $5 \mathrm{P}$ fit applied to a moving window of three-day sun interference collection (Frech 2009) might be more appropriate, in favor of the accuracy of the method.

Acknowledgments. Thanks are given to the DRAO (National Research Council Canada) for providing solar power measurements and to two anonymous reviewers who helped to improve the final form of this paper. This research was carried out in the framework of the Industrial Doctorate Projects, partly funded by the autonomous government of the Generalitat de Catalunya. 


\section{REFERENCES}

Blahak, U., 2008: An approximation to the effective beam weighting function for scanning meteorological radars with an axisymmetric antenna pattern. J. Atmos. Oceanic Technol., 25, 1182-1196, doi:10.1175/2007JTECHA1010.1.

Caruana, R. A., R. B. Searle, T. Heller, and S. I. Shupack, 1986: Fast algorithm for the resolution of spectra. Anal. Chem., $\mathbf{5 8}$ 1162-1167, doi:10.1021/ac00297a041.

Collier, C. G., 2009: On the propagation of uncertainty in weather radar estimates of rainfall through hydrological models. $\mathrm{Me}$ teor. Appl., 16, 35-40, doi:10.1002/met.120.

Darlington, T., M. Kitchen, J. Sugier, and J. de Rohan-Truba, 2003: Automated real-time monitoring of radar sensitivity and antenna pointing accuracy. 31st Int. Conf. on Radar Meteorology, Seattle, WA, Amer. Meteor. Soc., 7B.6. [Available online at https://ams.confex.com/ams/32BC31R5C/techprogram/paper_ 63543.htm.]

Doviak, R. J., and D. S. Zrnić, 2006: Doppler Radar and Weather Observations. 2nd ed. Dover Publications, 592 pp.

Eastment, J. D., J. W. F. Goddard, G. J. Davies, D. N. Ladd, and K. J. Twort, 2001: Boresight pointing calibration for the Chilbolton radar using galactic radio sources and satellite targets. Preprints, 30th Int. Conf. on Radar Meteorology, Munich, Germany, Amer. Meteor. Soc., P1.2. [Available online at https:// ams.confex.com/ams/30radar/techprogram/paper_19854.htm.]

Frech, M., 2009: Towards monitoring and assessing DualPol data quality. Evaluation of new technologies, OPERA III, Work Package 1.4a, EUMETNET, 2-54. [Available online at http:// www.eumetnet.eu/sites/default/files/OPERA_2009_02_WP1.4a_ Deliverable.pdf.]

_ 2013: Monitoring the data quality of the new polarimetric weather radar network of the German Meteorological Service. 36th Conf. on Radar Meteorology, Breckenridge, CO, Amer. Meteor. Soc., 9B.3. [Available online at https://ams.confex. com/ams/36Radar/webprogram/Paper228472.html.]

Friedrich, K., M. Hagen, and T. Einfalt, 2006: A quality control concept for radar reflectivity, polarimetric parameters, and Doppler velocity. J. Atmos. Oceanic Technol., 23, 865-887, doi:10.1175/JTECH1920.1.

Frush, C. L., 1984: Using the sun as calibration aid in multiple parameter meteorological radar. Preprints, 22nd Conf. on Radar Meteorology, Zurich, Switzerland, Amer. Meteor. Soc., 306-311.

Genz, A. C., and A. A. Malik, 1980: Remarks on algorithm 006: An adaptive algorithm for numerical integration over an $\mathrm{N}$ dimensional rectangular region. J. Comput. Appl. Math., 6, 295-302, doi:10.1016/0771-050X(80)90039-X.

Gourley, J. J., P. Tabary, and J. P. du Chatelet, 2006: Data quality of the Meteo-France C-band polarimetric radar. J. Atmos. Oceanic Technol., 23, 1340-1356, doi:10.1175/ JTECH1912.1.

Guo, H., 2011: A simple algorithm for fitting a Gaussian function. IEEE Signal Process. Mag., 28, 134-137, doi:10.1109/ MSP.2011.941846.

Harrison, D. L., S. J. Driscoll, and M. Kitchen, 2000: Improving precipitation estimates from weather radar using quality control and correction techniques. Meteor. Appl., 7, 135-144, doi:10.1017/S1350482700001468.

Holleman, I., 2005: Quality control and verification of weather radar wind profiles. J. Atmos. Oceanic Technol., 22, 15411550, doi:10.1175/JTECH1781.1.

_ and H. Beekhuis, 2004: Weather radar monitoring using the sun. KNMI Tech. Rep. TR-272, 40 pp.
— , and A. Huuskonen, 2013: Analytical formulas for refraction of radiowaves from exoatmospheric sources. Radio Sci., 48, 226-231, doi:10.1002/rds.20030.

- H. van Gasteren, and W. Bouten, 2008: Quality assessment of weather radar wind profiles during bird migration. J. Atmos. Oceanic Technol., 25, 2188-2198, doi:10.1175/2008JTECHA1067.1.

-, A. Huuskonen, M. Kurri, and H. Beekhuis, 2010: Operational monitoring of weather radar receiving chain using the sun. J. Atmos. Oceanic Technol., 27, 159-166, doi:10.1175/2009JTECHA1213.1.

Huuskonen, A., and I. Holleman, 2007: Determining weather radar antenna pointing using signals detected from the sun at low antenna elevations. J. Atmos. Oceanic Technol., 24, 476-483, doi:10.1175/JTECH1978.1.

—, M. Kurri, and H. Hohti, 2010: The use of the solar monitoring data to assess the quality and the stability of weather radar antenna systems. TECO-2010: WMO Tech. Conf. on Meteorological and Environmental Instruments and Methods of Observation, Helsinki, Finland, World Meteorological Organization, P2(9). [Available online at https://www.wmo.int/ pages/prog/www/IMOP/publications/IOM-104_TECO-2010/ P2_9_Huuskonen_Finland.pdf.]

,,,--- H. Beekhuis, H. Leijnse, and I. Holleman, 2014: Radar performance monitoring using the angular width of the solar image. J. Atmos. Oceanic Technol., 31, 1704-1712, doi:10.1175/JTECH-D-13-00246.1.

Joe, P., 2011: The WMO radar QC QPE Inter-Comparison Project RQQI. World Meteorological Organization Doc. CIMO/ IOC-RQQI -1/Doc. 2, 17 pp.

Lakshmanan, V., A. Fritz, T. Smith, K. Hondl, and G. Stumpf, 2007: An automated technique to quality control radar reflectivity data. J. Appl. Meteor. Climatol., 46, 288-305, doi:10.1175/JAM2460.1

Leskinen, M., P. Puhakka, and T. Puhakka, 2002: A method for estimating antenna beam parameters using the sun. Proceedings of the Second European Conference on Radar Meteorology, Copernicus Gesellschaft, 318-323.

Michelson, D., T. Einfalt, I. Holleman, U. Gjertsen, K. Friedrich, G. Haase, M. Lindskog, and A. Jurczyk, 2004: Weather radar data quality in Europe: Quality control and characterization. COST-717 Working Doc. WDF_MC 200403_1, 92 pp.

Muth, X., M. Schneebeli, and A. Berne, 2012: A sun-tracking method to improve the pointing accuracy of weather radar. Atmos. Meas. Tech., 5, 547-555, doi:10.5194/amt-5-547-2012.

Ośródka, K., J. Szturc, and A. Jurczyk, 2014: Chain of data quality algorithms for 3-D single-polarization radar reflectivity (RADVOL-QC system). Meteor. Appl., 21, 256-270, doi:10.1002/met.1323.

Pratt, J. F., and D. G. Ferraro, 1989: Automated solar gain calibration. Preprints, 24th Conf. on Radar Meteorology, Tallahassee, FL, Amer. Meteor. Soc., 619-622.

Probert-Jones, J. R., 1962: The radar equation in meteorology. Quart. J. Roy. Meteor. Soc., 88, 485-495, doi:10.1002/ qj. 49708837810.

Reimann, C., P. Filzmoser, R. G. Garrett, and R. Dutter, 2008: Statistical Data Analysis Explained: Applied Environmental Statistics with R. 3rd ed. John Wiley and Sons Ltd., 362 pp.

Rossa, A., and Coauthors, 2010: Propagation of uncertainty from observing systems into NWP: COST-731 Working Group 1. Atmos. Sci. Lett., 11, 145-152, doi:10.1002/asl.274.

Saltikoff, E., A. Huuskonen, H. Hohti, J. Koistinen, and H. Järvinen, 2010: Quality assurance in the FMI Doppler weather radar network. Boreal Environ. Res., 15, 579-594. 
Sprent, P., and N. C. Smeeton, 2007: Applied Nonparametric Statistical Methods. 4th ed. Chapman and Hall/CRC, 530 pp.

Szturc, J., K. Ośródka, and A. Jurczyk, 2011: Quality index scheme for quantitative uncertainty characterization of radar-based precipitation. Meteor. Appl., 18, 407-420, doi:10.1002/met.230.

Tapping, K., 2001: Antenna calibration using the $10.7 \mathrm{~cm}$ solar flux. Workshop on Radar Calibration, Albuquerque, NM, Amer. Meteor. Soc., 32 pp.
Vaisala, 2014: User's manual: Digital IF receiver/Doppler signal processor, RVP8. Vaisala Oyj Doc. M211321EN-D, 522 pp.

Whiton, R. C., P. L. Smith, and A. C. Harbuck, 1976: Calibration of weather radar systems using the sun as a radio source. Preprints, 17th Conf. on Radar Meteorology, Seattle, WA, Amer. Meteor. Soc., 60-65.

WMO, 2008: Guide to meteorological instruments and methods of observation. 7th ed. World Meteorological Organization Doc. WMO-8, 681 pp. 